\title{
Higher Order Approximations to the Boundary Conditions for the Finite Element Method
}

\author{
By J. J. Blair*
}

\begin{abstract}
We consider here the approximation of essential boundary conditions for the finite element solutions of second order elliptic equations in two dimensions. Nonhomogeneous boundary conditions on curved boundaries are treated.

The approach is to use trial functions which interpolate (in a generalized sense) functions satisfying the boundary conditions. The work is directed to showing in what manner this interpolation should be done to achieve the maximum accuracy and computational simplicity. These methods can be used to construct approximations of arbitrary high order of accuracy. Several examples are given.
\end{abstract}

1. Introduction. We consider here the approximate solution by the finite element method of the Dirichlet problem for second order elliptic equations in two dimensions. Nonhomogeneous boundary conditions on general smooth domains are treated. The case in which the boundary conditions are homogeneous and the domain is polygonal has received much study (cf. [10], [11], [14], [16] and [17]). A natural approach to problems on smooth domains is to replace the domain with a polygonal approximation; however, this approach has limited order of accuracy. The error, in the $H^{1}$. norm, is at best $O\left(h^{3 / 2}\right)$ and in the $L^{2}$-norm $O\left(h^{2}\right)$ (cf. [4], [15]). The parameter $h$ is the "mesh size".

Another approach, taken by Berger, Scott and Strang in [3], is to approximate the boundary conditions. Specifically, they take as trial functions piecewise polynomials which interpolate smooth functions satisfying the boundary conditions. This means that the trial functions and, perhaps, some of their derivatives agree, at a finite set of points on the boundary, with functions which satisfy the boundary conditions.

Our approach is very similar. The difference is that we use the term interpolate in a wider sense. We use as trial functions piecewise polynomials whose weighted averages over segments of the boundary are equal to the same averages of functions which satisfy the boundary conditions. We show that by using polynomial weights of the appropriate degree one obtains methods of arbitrary high order of accuracy and minimal complexity. As an example we show that using piecewise cubics and appropriate approximate boundary conditions the error is $O\left(h^{3}\right)$ in the $H^{1}$-norm and $O\left(h^{4}\right)$ in the $L^{2}$-norm. This method has the same complexity (a term made precise later) as the simplest method utilizing cubics on a polygonal domain.

Bramble and Schatz ([8], [9]) have also introduced a method -which eliminates

Received February 9, 1973; revised January 20, 1974.

AMS (MOS) subject classifications (1970). Primary 65N15, 65N30, 35A40.

* This research was supported in part by the Office of Naval Research under Contract N0014-69-A0200-1017 at the University of California, Berkeley.

Copyright $\odot$ 1976, American Mutnumutical Society 
the necessity of using trial functions which satisfy the boundary conditions. Their method, like ours, has the optimal order of accuracy consistent with the approximation properties of the trial functions. Their methods, however, have greater computational complexity due to the fact that they require a Ritz approximation to the solution of a fourth order equation. Solving a fourth order equation causes additional difficulties for two reasons: (1) The condition number of the system of equations to be solved is larger. (2) Smoother $\left(C^{1}\right)$ trial functions are required. Bramble and Nitsche introduced a method in [6] which, while still requiring the computation of the Ritz approximation of the solution of a fourth order equation, eliminates the first difficulty mentioned above, because the fourth order term is multiplied by a small parameter. The second difficulty, however, remains.

Bramble, Dupont and Thomée give a method in [7] which yields the same order of accuracy as the method of this paper. Their method, though stated quite differently, is very similar to ours.

2. The General Theory. We shall consider the problem of approximating the solution $u$ to the problem

$$
L u=f \quad \text { in } \Omega, \quad u=g \quad \text { on } \Gamma,
$$

where $\Omega$ is a bounded open subset of the plane, $\Gamma$ is its smooth $\left(C^{\infty}\right)$ boundary, $f$ and $g$ are sufficiently smooth functions on $\Omega$ and $\Gamma$, and $L$ is a uniformly elliptic selfadjoint differential operator of second order, i.e.

$$
L u=-\sum_{i, j} D_{i} a_{i j} D_{j} u+c u
$$

with $a_{i j}$ a symmetric positive definite matrix whose smallest eigenvalue is uniformly bounded away from zero. We shall assume that the coefficients are all bounded real valued $C^{\infty}$ functions and, in fact, that all functions which appear are real valued. The selfadjointness plays no essential role whatsoever, but it does simplify the exposition by allowing familiar words (norm, scalar product, orthogonal, etc.) to be used in place of expressions.

We shall denote by $H^{k}(\Omega)$ the Hilbert space of functions whose derivatives up to order $k$ are in $L^{2}(\Omega)$. We use the usual norm on $H^{k}(\Omega)$ given by

$$
\|u\|_{k, \Omega}^{2}=\sum_{|\alpha| \leqslant k}\left\|D^{\alpha} u\right\|_{0, \Omega}^{2}=\sum_{\alpha_{1}+\alpha_{2} \leqslant k}\left\|D_{1}^{\alpha} D_{2}^{\alpha} u\right\|_{0, \Omega}^{2},
$$

where $\|\cdot\|_{0, \Omega}$ is the usual norm on $L^{2}(\Omega)$.

We associate with $L$ the bilinear form

$$
a(u, v)=\sum_{i, j}\left(D_{i} v, a_{i j} D_{j} u\right)+(c u, v)
$$

where $(\cdot, \cdot)$ denotes the scalar product on $L^{2}(\Omega)$. We assume that

$$
\begin{gathered}
|a(u, v)| \leqslant E\|u\|_{1, \Omega}\|v\|_{1, \Omega} \quad \text { for all } u, v \in H^{1}(\Omega), \text { and } \\
a(v, v) \geqslant e\|v\|_{1, \Omega}^{2} \quad \text { for all } v \in H_{0}^{1}(\Omega),
\end{gathered}
$$

where $e$ is positive constant and $H_{0}^{1}(\Omega)$ consists of those functions in $H^{1}(\Omega)$ which vanish on $\Gamma$. We let 


$$
\|v\|_{a}^{2}=a(v, v) .
$$

From the second half of (2) we see that, restricted to $H_{0}^{1}(\Omega),\|\cdot\|_{a}$ is a norm equivalent to $\|\cdot\|_{1, \Omega}$. We will call $\|\cdot\|_{a}$ the energy norm (even when applied to functions not in $\left.H_{0}^{1}(\Omega)\right)$.

We will denote by $\langle\cdot, \cdot\rangle$ the scalar product on $L^{2}(\Gamma)$. The integral is understood to be with respect to arc length. In fact we shall implicitly assume throughout the paper that each connected component of $\Gamma$ is parametrized by the arc length $t$, along that component. Thus, when we say a function on $\Gamma$ is a polynomial on some segment of $\Gamma$, we mean that it is a polynomial in the variable $t$.

It is well known that Eq. (1) is equivalent to

$$
a(u, \phi)=(f, \phi) \quad \forall \phi \in H_{0}^{1}(\Omega), \gamma u=g,
$$

where $\gamma$ is the trace operator on $H^{1}(\Omega)$, i.e. $\gamma u=$ the restriction of $u$ to $\Gamma$. We shall often omit the symbol $\gamma$ when it is felt that no confusion will arise. The general approximation method we study here consists of selecting a subspace $F^{\boldsymbol{h}}$ of $H^{\mathbf{1}}(\Omega)$ and a space $S^{h}$ of distributions on $\Gamma$ and letting the approximation, $\hat{u}$, of $u$ be the function in $F^{h}$ which satisfies

$$
a(\hat{u}, \phi)=(f, \phi) \quad \forall \phi \in F_{0}^{h}, \gamma \hat{u}-g \perp S^{h} .
$$

The last condition means that $\langle\gamma u, \psi\rangle=\langle g, \psi\rangle$ for all $\psi \in S^{h}$, and $F_{0}^{h}$ consists of those functions in $F^{h}$ which are orthogonal to $S^{h}$ on $\Gamma$ (i.e. $\phi \in F_{0}^{h}$ iff $\phi \in F^{h}$ and $\langle\gamma \phi, \psi\rangle=$ $0 \forall \psi \in S^{h}$ ). Since we have in mind the finite element method, i.e. $F^{h}$ consists of functions which are polynomials on "elements" of $\Omega$, we have introduced the positive parameter $h$ which will later be used as the diameter of the elements.

The approximation method, (4), is similar to the classical Ritz method, the difference being that all boundary conditions are approximated. The fact that the approximation $\hat{u}$ does not satisfy the boundary conditions of the problem is relatively minor. The important point is that the test functions, $\phi$, do not vanish on $\Gamma$. It is this fact which spoils the best approximation property characteristic of the classical Ritz method.

In order for this method to be of any value, there must exist a unique solution to the pair of equations in (4). We shall make the assumption that for some positive $e$,

$$
a(v, v) \geqslant e\|v\|_{1}^{2} \quad \text { for all } v \in F_{0}^{h},
$$

which guarantees uniqueness. In many cases it is quite easy to verify this directly. We point out (without proof) that (5) will always be satisfied (for sufficiently small $h$ ) if the space $S^{h}$ has the uniform $A$-property of order 0 , which will be defined in Section 3. All of the spaces we construct in Section 5 have this property. The existence of a solution is guaranteed by (5) and two additional assumptions. The first is that $F_{0}^{h}$ is closed in $H^{\mathbf{1}}(\Omega)$, which is always true if $F^{h}$ has finite dimension. The second is that there is a function in $F^{h}$ which satisfies the approximate boundary conditions $\left(\gamma u-g \perp S^{h}\right)$. It is, of course, tacitly assumed that the function $g$ and all functions $v \in F^{h}$ are smooth enough that the linear functionals $\langle g, \psi\rangle$ and $\langle\gamma, \psi\rangle$ are defined for all $\psi \in S^{h}$. 
We now turn to the problem of estimating the error in this approximation method. We first introduce the auxiliary function $\tilde{u} \in F^{h}$ satisfying

$$
a(\tilde{u}, \phi)=a(u, \dot{\phi}) \quad \forall \phi \in F_{0}^{h}, \gamma \tilde{u}-g \perp S^{h} .
$$

If $v$ is any function in $F^{h}$ which satisfies $\gamma v-g \perp S^{h}$, then $\|u-\tilde{u}\|_{a} \leqslant\|u-v\|_{a}$. To show this we note that since $a(u-\tilde{u} \phi)=0$ for all $\phi \in F_{0}^{h}$, we have

$$
a(u-\tilde{u}, u-\tilde{u})=a(u-\tilde{u}, u-\tilde{u})+a(u-\tilde{u}, \tilde{u}-v),
$$

or

$$
\|u-\tilde{u}\|_{a}^{2}=a(u-\tilde{u}, u-v)
$$

Using the Schwarz inequality,

$$
\|u-\tilde{u}\|_{a}^{2} \leqslant\|u-\tilde{u}\|_{a}\|u-v\|_{a}
$$

and dividing by $\|u-\tilde{u}\|_{a}$ give the result.

Thus, we see that $\widetilde{u}$, although it does not satisfy the boundary conditions, has the best approximation property which is characteristic of a true Ritz approximation. Also, since $u-\tilde{u}$ is orthogonal (with respect to the energy scalar product) to $F_{0}^{h}$ and $\tilde{u}-\hat{u} \in F_{0}^{h}$ we have

$$
\|u-\hat{u}\|_{a}^{2}=\|u-\tilde{u}\|_{a}^{2}+\|\tilde{u}-\hat{u}\|_{a}^{2} .
$$

We shall obtain bounds for each term on the right-hand side of (7) separately. Note that since this is an equality, a lower bound on either term yields a lower bound for the error.

The obtaining of a bound for $\|u-\tilde{u}\|_{a}$ is a standard approximation theory problem which we will not treat in any detail until Section 5 . To estimate $\tilde{u}-\hat{u}$ we introduce the Green's formula

$$
a(u, v)=(L u, v)+\langle\delta u, \gamma v\rangle
$$

which is valid for any $u$ and $v$ with $u \in H^{2}(\Omega)$ and $v \in H^{1}(\Omega)$. This is easily established by integrating the expression for $a$ by parts, which also yields

$$
\delta u=\sum_{i, j} v_{i} a_{i j} D_{j} u
$$

where $\left(\nu_{1}, \nu_{2}\right)$ is the unit (outward) normal to $\Gamma$. Using the definition (6) of $\tilde{u}$ and Green's formula, we have

$$
a(\tilde{u}, \phi)=a(u, \phi)=(f, \phi)+\langle\delta u, \gamma \phi\rangle \quad \forall \phi \in F_{0}^{h} .
$$

From the definition, (4), of $\hat{u}$,

$$
a(\hat{u}, \phi)=(f, \phi) \quad \forall \phi \in F_{0}^{h}
$$

Subtracting, we have

$$
a(\widetilde{u}-\hat{u}, \phi)=\langle\delta u, \gamma \phi\rangle \quad \forall \phi \in F_{0}^{h} .
$$


Since $\tilde{u}-\hat{u} \in F_{0}^{h}$, we have

$$
\|\tilde{u}-\hat{u}\|_{a}=\sup _{\phi \in F_{0}^{h} ;\|\phi\|_{a} \leqslant 1}\langle\delta u, \gamma \phi\rangle .
$$

Thus, we see that the error separates into two distinct parts, an approximation term $u-\tilde{u}$ and a boundary term $\tilde{u}-\hat{u}$. The boundary term (9) depends only on the properties of elements of $F_{0}^{h}$ on $\Gamma$.

To obtain the appropriate estimates for the boundary term we must introduce the Hilbert spaces $H^{s}(\Gamma)$ (for arbitrary real $s$ ) of distributions on $\Gamma$. If $s$ is a nonnegative integer, $H^{s}(\Gamma)$ consists of all functions on $\Gamma$ whose derivatives, up to order $s$, are in $L^{2}(\Gamma)$. For nonintegral $s, H^{s}(\Gamma)$ is an interpolation space between spaces with integral $s$; and $H^{-s}(\Gamma)$ is the dual of $H^{s}(\Gamma)$. We refer the reader to Chapter 1 of [12] for the details. We note that since $H^{-s}(\Gamma)$ is the dual of $H^{s}(\Gamma)$, we have the generalized Schwarz inequality $\langle u, v\rangle \mid \leqslant\|u\|_{s, \Gamma}\|v\|_{-s, \Gamma}$ which is valid for all $s$. We also have from the trace theorem (e.g. [12, Theorem 9.4]) that for $p>3 / 2, \delta$ is a bounded operator from $H^{p}(\Omega)$ onto $H^{p-3 / 2}(\Gamma)$, and $\gamma$ is a bounded operator from $H^{p-1}(\Omega)$ onto $H^{p-3 / 2}(\Gamma)$. We obtain immediately from (9), the trace theorem and the Schwarz inequality that

$$
\|\tilde{u}-\hat{u}\|_{a} \leqslant C\|u\|_{p, \Omega} \sup _{\phi \in F_{0}^{h} ;\|\phi\|_{a} \leqslant 1}\|\gamma \phi\|_{3 / 2-p, \Gamma},
$$

where $C=C(p, \Omega, L)$ is a constant.

We wish to analyze the rate at which the right-hand side of (10) goes to zero as $h$ goes to zero. For this we must assume that the pair $\left(F^{h}, S^{h}\right)$ belongs to some fixed family $(F, S)$ of spaces. We shall use $C$ as a generic constant which does not necessarily have the same value at each occurence.

Definition 1. The pair $\left(F^{h}, S^{h}\right)$ is said to have the approximation property of order $p$ if for every $v \in H^{p}(\Omega)$ there is a $\widetilde{v} \in F^{h}$ satisfying

$$
\gamma(v-\widetilde{v}) \perp S^{h} \text { and }\|v-\widetilde{v}\|_{1, \Omega} \leqslant C h^{k-1}\|v\|_{k, \Omega},
$$

where $C=C(p, \Omega)$ and $2 \leqslant k \leqslant p$. An operator $P$ from $H^{p}(\Omega)$ into $F^{h}$ with the property that $P v$ can be substituted for $\widetilde{v}$ above will be called an approximation operator of order $p$ for $\left(F^{h}, S^{h}\right)$.

Definition 2. The space $S^{h}$ is said to have the weak A-property of order $q$ with respect to $F^{h}$ if there is a $C=C(q, \Omega)$ such that

$$
\sup _{\phi \in F_{0}^{h} ;\|\phi\|_{1, \Omega} \leqslant 1}\|\gamma \phi\|_{-s, \Gamma} \leqslant C h^{s+1 / 2} \text { for } 0 \leqslant s \leqslant q .
$$

THEOREM 1. If the pair $\left(F^{h}, S^{h}\right)$ has the approximation property of order $p, S^{h}$ has the weak A-property of order $p-3 / 2$ (with respect to $F^{h}$ ) and the solution $u$ of (3) belongs to $H^{p}(\Omega)$, then

$$
\|u-\hat{u}\|_{1, \Omega} \leqslant C\|u\|_{p, \Omega} h^{p-1},
$$

where $\hat{u}$ is the solution of (4), $C=C(p, \Omega, L)$ and $p \geqslant 2$.

Proof. Since $\left(F^{h}, S^{h}\right)$ has the approximation property of order $p$, there is a $u^{*} \in F^{h}$ satisfying $\gamma\left(u-u^{*}\right) \perp S^{h}$ and 


$$
\left\|u-u^{*}\right\|_{1, \Omega} \leqslant C h^{p-1}\|u\|_{p, \Omega} .
$$

We then have for the function $\tilde{u}$ defined in (6),

$$
\|u-\tilde{u}\|_{1, \Omega} \leqslant E\|u-\tilde{u}\|_{a} \leqslant E\left\|u-u^{*}\right\|_{a} \leqslant(E / e)\left\|u-u^{*}\right\|_{1, \Omega},
$$

where the inequalities follow from (2), the best approximation property of $\tilde{u}$ and (5), respectively. Thus, we have the bound for $\|u-\tilde{u}\|_{1, \Omega}$. The bound for $\|\tilde{u}-\hat{u}\|_{1, \Omega}$ follows directly from Definition 2, (10) and the equivalence of the energy and $H^{1}(\Omega)$ norms on $F_{0}^{h}$.

We should point out that since $\delta$ maps $H^{p}(\Omega)$ onto $H^{p-3 / 2}(\Gamma)$ and the error equation (9) is an equality, the weak $A$-property of order $p-3 / 2$ is necessary for the error estimate of Theorem 1 to hold.

3. On the $A$-Property. We introduce here stronger forms of the $A$-property which will be used later.

Definition 3. The space $S^{h}$ is said to have the strong A-property of order $q$ with respect to $F^{h}$ if there is a $C=C(q, \Omega)$ such that

$$
\sup _{\phi \in F_{0}^{h} ;\|\gamma \phi\|_{1 / 2, \Gamma} \leqslant 1}\|\gamma \phi\|_{-s, \Gamma} \leqslant C h^{s+1 / 2} \text { for } \overline{0} \leqslant s \leqslant q .
$$

In this definition (unlike Definition 2) no reference is made to the behavior of $\phi$ in the interior of $\Omega$. The theorem below gives a useful relationship between the strong and weak $A$-properties.

THEOREM 2. If $S^{h}$ has the strong A-property of order $q$ with respect to $F^{h}$, and $F_{0}^{h}$ has the property that

$$
\|\gamma \phi\|_{1 / 2, \Gamma} \leqslant C h^{\rho}\|\phi\|_{1, \Omega}
$$

for all $\phi \in F_{0}^{h}$ and some $\rho \geqslant 0$, then $S^{h}$ has the weak A-property of order $q+\rho$ with respect to $F^{h}$.

Proof. Since $S^{h}$ has the strong $A$-property

$$
\|\gamma \phi\|_{-s, \Gamma} \leqslant C h^{s+1 / 2}\|\gamma \phi\|_{1 / 2, \Gamma} \leqslant C h^{s+\rho+1 / 2}\|\phi\|_{1, \Omega} \quad \text { for } \phi \in F_{0}^{h} \text { and } 0 \leqslant s \leqslant q,
$$

the last inequality following from the hypothesis. The result follows from the fact that

$$
\|\gamma \phi\|_{-(s+\rho), \Gamma} \leqslant C\|\gamma \phi\|_{-s, \Gamma} .
$$

Since $\gamma$ is a bounded operator from $H^{1}(\Omega)$ onto $H^{1 / 2}(\Gamma)$, the inequality in the statement of the theorem always holds for $\rho=0$; and therefore, the strong $A$-property implies the weak $A$-property of the same order.

Definition 4. The space $S^{h}$ is said to have the uniform A-property of order $q$ if $S^{h} \cap H^{-1 / 2}(\Gamma)$ has the strong A-property of order $q$ with respect to $H^{1}(\Omega)$.

This means that if $\psi$ is any function in $H^{1 / 2}(\Gamma)$ and $\psi \perp S^{h} \cap H^{-1 / 2}(\Gamma)$, then

$$
\|\psi\|_{-s, \Gamma} \leqslant C h^{s+1 / 2}\|\psi\|_{1 / 2, \Gamma} \text { for } 0 \leqslant s \leqslant q .
$$


If $S^{h}$ has the uniform $A$-property, then it has the strong $A$-property (of the same order) with respect to any subspace of $H^{1}(\Omega)$.

THEOREM 3. If $S^{h}$ has a subspace $S_{0}^{h} \subset L^{2}(\Gamma)$ with the property that for any $v \in H^{q}(\Gamma)$, there is a $\tilde{v} \in S_{0}^{h}$ satisfying $\|v-\widetilde{v}\|_{0, \Gamma} \leqslant C h^{q}\|v\|_{q, \Gamma}$ then $S^{h}$ has the uniform A-property of order $q$.

Proof. We let $P$ be the orthogonal projection operator (in $L^{2}(\Gamma)$ ) onto $S_{0}^{h}$ and $Q=I-P(I=$ the identity $)$. The hypothesis of the theorem is that

$$
\|Q v\|_{0, \Gamma} \leqslant C h^{q}\|v\|_{q, \Gamma}
$$

We wish to show first that if $0 \leqslant s, t \leqslant q$ then

$$
\|Q v\|_{-s, \Gamma} \leqslant C h^{s+t}\|v\|_{t, \Gamma} .
$$

From (11) this holds with $(s, t)=(0, q)$; and since $Q$ is a bounded operator (with norm 1) on $H^{0}(\Gamma),(12)$ holds with $(s, t)=(0,0)$. From the inequality for $s=0$ and $t=0$ or $q$ we obtain, by interpolation (cf. [5, Corollary 1] or [13, Theorem 3.1]), the same inequality for $s=0$ and $t \in[0, q]$, i.e.,

$$
\|Q v\|_{0, \Gamma} \leqslant C h^{r}\|v\|_{r, \Gamma} \text { for } 0 \leqslant r \leqslant q .
$$

To obtain the inequality (12) for positive $s$ note that

$$
\|Q v\|_{-s, \Gamma}=\sup _{\|\phi\|_{s, \Gamma} \leqslant 1}\langle Q v, \phi\rangle
$$

and that for any $\phi \in H^{s}(\Gamma)$,

$$
\langle Q v, \phi\rangle=\langle Q v, Q \phi\rangle \leqslant\|Q v\|_{0, \Gamma}\|Q \phi\|_{0, \Gamma} \leqslant C\|v\|_{t, \Gamma}\|\phi\|_{s, \Gamma} h^{s+t} .
$$

The last inequality is obtained by applying (13) for $r=s$ and $r=t$. Taking the sup over $\|\phi\|_{s, \Gamma} \leqslant 1$ establishes (12) for arbitrary $(s, t) \in[0, q] \times[0, q]$.

To complete the proof of the theorem we note that $v \perp S^{h}$ implies $v \perp S_{0}^{h}$ which means that $v=Q v$ and hence (from (12)) that

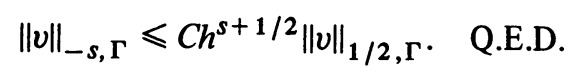

COROLlARY. Let $\Gamma$ be expressed as the union of nonoverlapping intervals of length $\leqslant h$. If $S^{h}$ contains all functions which are polynomials (in the arc length variable) of degree $\leqslant q-1(q \geqslant 1)$ on each interval, then $S^{h}$ has the uniform A-property of order $q$.

3. $L^{2}$ Error Estimates. For true Ritz approximations (i.e. when the trial functions satisfy the boundary conditions) the error in the $L^{2}$-norm is smaller than the error in the $H^{1}$-norm. For finite element methods, where $h$ is the maximum diameter of the elements, the error in the $L^{2}$-norm is $O\left(h^{p}\right)$ when the error in the $H^{1}$-norm is $O\left(h^{p-1}\right)$. We show below that this remains true for the class of methods considered in this paper under a variety of conditions on $\left(F^{h}, S^{h}\right)$. We will need first the following result which is a special case of Theorem 6.6 of [12].

Lemma. Let $v \in H^{1}(\Omega)$ satisfy. $L v=0$; then $\|v\|_{0, \Omega} \leqslant C\|v\|_{-1 / 2, \Gamma}$. 
Proof. Let $\phi$ be an arbitrary function in $H^{2}(\Omega) \cap H_{0}^{1}(\Omega)$; from the Green's formula (8) we have

$$
a(v, \phi)=(L v, \phi)-\langle\delta v, \gamma \phi\rangle=0
$$

the last equality due to the fact that $L v=0$ and $\gamma \phi=0$. Since $a(v, \phi)=a(\phi, v)$, we have

$$
0=a(\phi, v)=(L \phi, v)-\langle\delta \phi, \gamma\rangle\rangle
$$

which gives

$$
\begin{aligned}
(L \phi, v) & =\langle\delta \phi, \gamma v\rangle \leqslant\|\delta \phi\|_{1 / 2, \Gamma}\|v\|_{-1 / 2, \Gamma} \\
& \leqslant C\|\phi\|_{2, \Omega}\|v\|_{-1 / 2, \Gamma} \leqslant C\|L \phi\|_{0, \Omega}\|v\|_{-1 / 2, \Gamma}
\end{aligned}
$$

The last inequality follows from the regularity theorem for elliptic equations (cf. [1, Theorem 9.8] ; [12, Theorem 5.1]). Since $L$ maps $H^{2}(\Omega) \cap H_{0}^{1}(\Omega)$ onto $L^{2}(\Omega)$ we have

$$
\|v\|_{0, \Omega}=\sup _{\|L \phi\|_{0, \Omega} \leqslant 1}(L \phi, v) \leqslant C\|v\|_{-1 / 2, \Gamma},
$$

which completes the proof.

In the proof of the theorem below, we will denote the restriction of $L$ to $H_{0}^{1}(\Omega)$ by $B$. Note that $B$ is an isomorphism from $H_{0}^{1}(\Omega)$ onto $H^{-1}(\Omega)$, and that if $\phi \in L^{2}(\Omega)$, then $B^{-1} \phi \in H^{2}(\Omega) \cap H_{0}^{1}(\Omega)$ and

$$
\left\|B^{-1} \phi\right\|_{2, \Omega} \leqslant C\|\phi\|_{0, \Omega} .
$$

THEOREM 4. If in addition to the hypotheses of Theorem 1 we assume that:

(i) $S^{h}$ has the uniform A-property of order $1 / 2$, and or:

(iia) $S^{h}$ has the strong A-property of order $p-3 / 2$ with respect to $F^{h}$,

(iib) $S^{h}$ has the strong A-property of order $p-3 / 2-s$ with respect to $F^{h}$ and there is an approximation operator $P$ of order $p$ for $\left(F^{h}, S^{h}\right)$ with the property that

$$
\|\gamma P w\|_{1 / 2, \Gamma} \leqslant C h^{1+s}\|w\|_{2, \Omega} \quad \text { for all } w \in H^{2}(\Omega) \cap H_{0}^{1}(\Omega)
$$

then

$$
\|u-\hat{u}\|_{0, \Omega} \leqslant C h^{p}\|u\|_{p, \Omega}
$$

or if:

(iic) $u \in H^{p+1}(\Omega)$ and $S^{h}$ has the weak A-property of order $p-1 / 2$ with respect to $F^{h}$, then

$$
\|u-\hat{u}\|_{0, \Omega} \leqslant C h^{p}\|u\|_{p+1, \Omega}
$$

Proof. We let $u-\hat{u}=r$ and decompose $r$ as $r=r_{1}+r_{2}$ with

$$
L r_{1}=L r, \quad \gamma r_{1}=0 \quad \text { and } L r_{2}=0, \gamma r_{2}=\gamma r .
$$

We first derive the bound for $r_{2}$ : 


$$
\left\|r_{2}\right\|_{0, \Omega} \leqslant C\|\gamma r\|_{-1 / 2, \Gamma} \leqslant C h\|\gamma r\|_{1 / 2, \Gamma} \leqslant C h\|r\|_{1, \Omega} \leqslant C h^{p}\|u\|_{p, \Omega} .
$$

The first inequality follows from the lemma and the fact that $\gamma r_{2}=\gamma r$, the second because $r \perp S^{h}$ and $S^{h}$ has the uniform $A$-property of order 1/2, the third from the fact that $\gamma$ is a bounded operator from $H^{1}(\Omega)$ onto $H^{1 / 2}(\Gamma)$, and the fourth from Theorem 1.

We now obtain the bound for $r_{1}$. To do this we let $\phi$ be an arbitrary function in $L^{2}(\Omega)$ and show that $\left(r_{1}, \phi\right) \leqslant C h^{p}\|\phi\|_{0, \Omega}$. Since $\gamma r_{1}=0$, we have

$$
r_{1}=B^{-1} L r_{1}=B^{-1} L r .
$$

This gives us immediately that

$$
\left(r_{1}, \phi\right)=\left(B^{-1} L r, \phi\right)=\left(L r, B^{-1} \phi\right)=a\left(r, B^{-1} \phi\right) .
$$

The last equation holds because $B^{-1} \phi$ vanishes on $\Gamma$. We now let $P$ be an approximation operator of order $p$ for $\left(F^{h}, S^{h}\right)$ and $Q=I-P$. We write the equality above as

$$
\left(r_{1}, \phi\right)=a\left(r, Q B^{-1} \phi\right)+a\left(r, P B^{-1} \phi\right),
$$

and estimate each term on the right-hand side separately.

From (2) we have

$$
a\left(r, Q B^{-1} \phi\right) \leqslant E\|r\|_{1, \Omega}\left\|Q B^{-1} \phi\right\|_{1, \Omega} .
$$

From Theorem 1 we know that $\|r\|_{1, \Omega} \leqslant C h^{p-1}\|u\|_{p, \Omega}$. Also, since $\left\|B^{-1} \phi\right\|_{2, \Omega} \leqslant$ $C\|\phi\|_{0, \Omega}$ and $\left(F^{h}, S^{h}\right)$ has the approximation property of order 2, we have $\left\|Q B^{-1} \phi\right\|_{1, \Omega} \leqslant C h\|\phi\|_{0, \Omega}$. These results establish the appropriate bound for the first term in (14).

To estimate the second term in (14) we note that $P B^{-1} \phi \in F_{0}^{h}$ and recall that $r=u-\hat{u}$. For arbitrary $\psi \in H^{1}(\Omega)$ we have from Green's formula

$$
a(u, \psi)=(f, \psi)+\langle\delta u, \gamma \psi\rangle,
$$

where $f=L u$. From the definition of $\hat{u}$, we have

$$
a(\hat{u}, \psi)=(f, \psi) \text { for all } \psi \in F_{0}^{h} .
$$

Subtracting these two equations gives us

$$
a\left(r, P B^{-1} \phi\right)=\left\langle\delta u, \gamma P B^{-1} \phi\right\rangle .
$$

We assume now that condition (iib) is satisfied. From (15) we have

$$
a\left(r, P B^{-1} \phi\right) \leqslant\|\delta u\|_{p-3 / 2, \Gamma}\left\|\gamma P B^{-1} \phi\right\|_{3 / 2-p, \Gamma} .
$$

Since $\delta$ is a bounded operator from $H^{p}(\Omega)$ onto $H^{p-3 / 2}(\Gamma)$ and $S^{h}$ has the strong $A$-property of order $p-3 / 2-s$ with respect to $F^{h}$, the above yields

$$
a\left(r, P B^{-1} \phi\right) \leqslant C\|u\|_{p, \Omega} h^{p-1-s}\left\|\gamma P B^{-1} \phi\right\|_{1 / 2, \Gamma}
$$

To obtain the desired result we must show that $\left\|\gamma P B^{-1} \phi\right\|_{1 / 2, \Gamma} \leqslant C h^{1+s}\|\phi\|_{0, \Omega}$. 
This follows directly from the hypothesis and the regularity result immediately preceding the statement of the theorem.

If (iia) is satisfied, then (16) holds for $s=0$; and we must show that $\left\|\gamma P B^{-1} \phi\right\|_{1 / 2, \Gamma} \leqslant C h\|\phi\|_{0, \Omega}$. Now, since $B^{-1} \phi$ vanishes on $\Gamma, \gamma P B^{-1} \phi=-\gamma Q B^{-1} \phi$; and therefore,

$$
\left\|\gamma P B^{-1} \phi\right\|_{1 / 2, \Gamma} \leqslant C\left\|Q B^{-1} \phi\right\|_{1, \Omega}
$$

The quantity on the right has already been shown to be bounded by $C h\|\phi\|_{0, \Omega}$ so the result is established.

If condition (iic) is satisfied, we have from (15),

$$
a\left(r, P B^{-1} \phi\right) \leqslant\|\delta u\|_{p-1 / 2, \Gamma}\left\|\gamma P B^{-1} \phi\right\|_{1 / 2-p, \Gamma} \leqslant C\|u\|_{p+1, \Omega} h^{p}\left\|P B^{-1} \phi\right\|_{1, \Omega} .
$$

Since $\left\|P B^{-1} \phi\right\|_{1, \Omega} \leqslant C\|\phi\|_{0, \Omega}$, the proof is complete.

We should point out that condition (i) is only used to establish that $\|\gamma(u-\hat{u})\|_{-1 / 2, \Gamma} \leqslant C h^{p}$. If the boundary data satisfy more than the minimal smoothness requirement (which is $g \in H^{p-1 / 2}(\Gamma)$ ), this result can often be obtained when condition (i) is not satisfied.

The usefulness of condition (iib) of the theorem was discovered by Berger, see [2].

5. Construction of Spaces. In this section we construct spaces which have the approximation property of order 3 and 4 and have the corresponding optimal order of accuracy in both the $H^{1}$-norm and $H^{0}$-norm. The spaces are slight modifications of the spaces of piecewise quadratics and cubics on a triangulation of $\Omega$ (cf. [3] , [10], $[14],[15],[16])$ which are commonly used in the finite element method on polygonal domains. The resulting equations which must be solved have the same computational complexity as in the corresponding methods which have been used in polygonal domains. By this we mean that the same number of unknowns must be solved for and that the matrices associated with the equations have nonzero entries in exactly the same places. The constructions given here, and the proofs of the optimal order of accuracy, extend very easily to the spaces of higher order polynomials presented by Bramble and Zlámal in [10].

In all of the constructions below we shall assume that $\Omega$ has been subdivided into triangles, the subdivision satisfying certain restrictions which we describe here. First of all, any two triangles in the subdivision which intersect are required to have either a common vertex or a common side; no other situations are allowed. Next, we assume that there is a fixed constant $\theta>0$ such that every angle of every triangle is greater than $\theta$. A triangle is allowed to have one curved side which coincides with a segment of $\Gamma$; otherwise, all sides are straight line segments. The parameter $h$ will denote the length of the longest side of any triangle in the subdivision.

5.1. Space no. 1. The space $F^{h}$ consists of all functions which are quadratic in each triangle and continuous on $\bar{\Omega}$. To describe $S^{h}$ we refer to the picture below of a triangle in the subdivision. The side $A B$ is a curved side on $\Gamma$. The space $S^{h}$ 


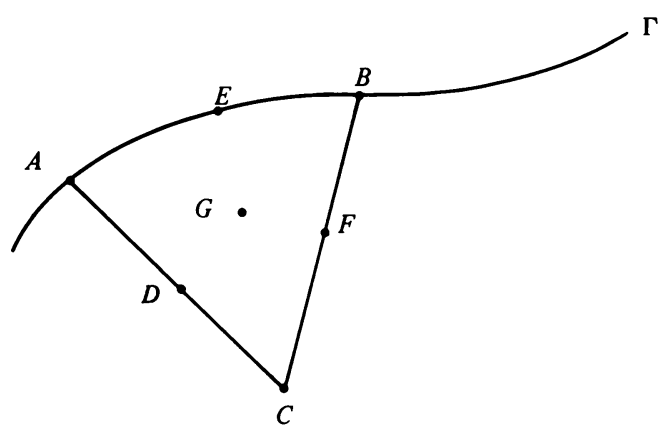

FIGURE 1

is the space spanned by delta-functions with support at vertices on $\Gamma$ (such as $A$ and $B$ ) and functions which are constant on each segment (such as $A B$ ) of $\Gamma$ which is the side of a triangle.

The pair $\left(F^{h}, S^{h}\right)$ has the approximation property of order 3 . This can be shown by constructing, for each $u \in H^{3}(\Omega)$, its interpolate, $u_{h}$, in $F^{h}$ and showing that the error $u-u_{h}$ has the correct asymptotic behavior in the $H^{1}(\Omega)$ - and $L^{2}(\Omega)$-norms. The interpolate of $u$ on a triangle with a side on $\Gamma$ is the polynomial of degree two which agrees with $u$ at $A, D, C, F$ and $B$ and which has the same average value as $u$ on the segment $A B$. On other triangles the interpolate is the quadratic which agrees with $u$ at the vertices and midpoints of the sides. The approximation result then follows from a slight modification of the arguments given by Bramble and Zlámal in [10].

From the corollary to Theorem 3 , the space $S^{h}$ has the strong $A$-property of order 1. Also, $S^{h}$ has the weak $A$-property of order 2. This follows from Theorem 2 and the inequality

$$
\|\phi\|_{1 / 2, \Gamma} \leqslant C h\|\phi\|_{1, \Omega},
$$

which is valid for all $\phi \in F_{0}^{h}$. This inequality can be derived by using a slight modification of the argument used by Berger, Scott and Strang in [3] (to derive their (13)) to obtain $\|\phi\|_{0, \Gamma} \leqslant C h^{3 / 2}\|\phi\|_{1, \Omega}$, using similar reasoning to obtain $\|\phi\|_{1, \Gamma} \leqslant C h^{1 / 2}\|\phi\|_{1, \Omega}$ and then interpolating to obtain (17).

Since $\left(F^{h}, S^{h}\right)$ has the approximation property of order 3 and the weak $A$-property of order $3 / 2$, we have, from Theorem 1 ,

$$
\|u-\hat{u}\|_{1, \Omega} \leqslant C h^{p-1}\|u\|_{p, \Omega} \text { for } 2 \leqslant p \leqslant 3 .
$$

To obtain the optimal error estimate in the $L^{2}$-norm we will need the inequality

$$
\|\widetilde{w}\|_{1 / 2, \Gamma} \leqslant C h^{3 / 2}\|w\|_{2, \Omega} \quad \forall w \in H^{2}(\Omega) \cap H_{0}^{1}(\Omega),
$$

where $\widetilde{w}$ is the interpolate of $w$ described previously. In order to derive (19) we will denote by $\Omega_{h}$ the union of the triangles, in the subdivision of $\Omega$, which intersect $\Gamma$ and note that (cf. [3] ) (17) holds with $\Omega$ replaced by $\Omega_{h}$, i.e.

$$
\|\widetilde{w}\|_{1 / 2, \Gamma} \leqslant C h\|\widetilde{w}\|_{1, \Omega}{ }_{h}
$$

Using the fact that $\left(F^{h}, S^{h}\right)$ has the approximation property of order 2 , we have

$$
\|\widetilde{w}\|_{1, \Omega_{h}} \leqslant\|\widetilde{w}-w\|_{1, \Omega_{h}}+\|w\|_{1, \Omega_{h}} \leqslant C h\|w\|_{2, \Omega}+\|w\|_{1, \Omega_{h}} .
$$


From [4, Lemma 3.2] we obtain $\|w\|_{1, \Omega} \leqslant C h^{1 / 2}\|w\|_{2, \Omega}$ for all $w \in H^{2}(\Omega) \cap$ $H_{0}^{1}(\Omega)$. Substituting this into (21) and the result into (20) gives (19).

The fact that $S^{h}$ has the uniform $A$-property of order 1 and the inequality (19) establish that $\left(F^{h}, S^{h}\right)$ satisfies the hypothesis (iib) of Theorem 4 with $p=3, s=1 / 2$ and $P$ being the operator of interpolation; hence,

$$
\|u-\hat{u}\|_{0, \Omega} \leqslant C h^{p}\|u\|_{p, \Omega} \text { for } 2 \leqslant p \leqslant 3 .
$$

5.2. Space no. 2. This pair of spaces has the approximation property of order 4 , the strong $A$-property of order 2 and the weak $A$-property of order 3 . The space $F^{h}$ is a slight modification of the commonly used (cf. [3], [10], [14], [16] ) space of piecewise cubics. This consists of all functions which are cubic on each triangle, continuous on $\bar{\Omega}$ and have continuous first derivatives at all vertices. The modification consists of relaxing the requirement of continuity of first derivatives at the vertices on the boundary, $\Gamma$. To describe this we again refer to Fig. 1, which shows a typical boundary triangle, and use point $A$ as a typical boundary vertex. Our relaxed continuity requirement is then as follows: The first derivative, in the direction $A C$, of the cubic defined in triangle $A B C$ equals the first derivative, in the same direction, of the cubic defined in the triangle which shares side $A C$ with triangle $A B C$. This continuity requirement allows discontinuities in the derivatives tangent to $\Gamma$ at the vertices on $\Gamma$.

The space $S^{h}$ consists of delta-functions at the vertices on $\Gamma$ and functions which are polynomials of degree one on each segment of $\Gamma$ which is a triangle side. From the corollary to Theorem $3, S^{h}$ has the strong $A$-property of order 2 with respect to $F^{h}$. One can show that $S^{h}$ has the weak $A$-property of order 3 with respect to $F^{h}$ by showing that $\|\phi\|_{1 / 2, \Gamma} \leqslant C h\|\phi\|_{1, \Omega}$ for all $\phi \in F_{0}^{h}$ and applying Theorem 2. The inequality above can be deduced by the same method discussed in Section 5.1 .

The proof that the pair $\left(F^{h}, S^{h}\right)$ has the approximation property of order 4 follows the standard procedure (cf. [10] ) of constructing, for any $u \in H^{4}(\Omega)$, an interpolate $u_{h} \in F^{h}$ which satisfies $u-u_{h} \perp S^{h}$, and showing that $u_{h}$ approximates $u$ with the required accuracy.

Since $\left(F^{h}, S^{h}\right)$ has the approximation property of order 4 and the weak $A$-property of order $5 / 2$, we have, from Theorem 1 ,

$$
\|u-\hat{u}\|_{1, \Omega} \leqslant C h^{p-1}\|u\|_{p, \Omega} \text { for } 2 \leqslant p \leqslant 4 .
$$

Using the same argument as in the previous section establishes that this pair of spaces satisfies condition (iib) of Theorem 4 (with $p=4, s=1 / 2$ ); and therefore,

$$
\|u-\hat{u}\|_{0, \Omega} \leqslant C h^{p}\|u\|_{p, \Omega} \text { for } 2 \leqslant p \leqslant 4 .
$$

5.3. Comments. The constructions given above easily extend to higher order polynomials. The general result is: If $F^{h}$ consists of polynomials of degree $p-1$ (with the appropriate continuity conditions), $S^{h}$ consists of delta-functions at the vertices on $\Gamma$ and polynomials of degree $p-3$ on the segments between the vertices; and the solution to the boundary value problem belongs to $H^{p-s}(\Omega)$, then the 1-norm of the error is $O\left(h^{p-1-s}\right)$ (for $\left.0 \leqslant s \leqslant p-2\right)$; and the $L^{2}$-norm of the error is $O\left(h^{p-s}\right)$ (for $0 \leqslant s \leqslant p-2$ ). 
An essential ingredient for this result is that, since $F^{h}$ consists of piecewise polynomials, $S^{h}$ has (with respect to $F^{h}$ ) the weak $A$-property of one order higher than it has the strong $A$-property. This idea, stated in different terms, was first utilized by Berger, Scott and Strang in [3].

Our space no. 1 was constructed because it is the simplest nontrivial example of a method which can be used to obtain any order of accuracy desired.

We should point out that there is a method which appears superior to using our space no. 1. Zlámal has shown in [17] that if one makes a slight modification of the usual space of piecewise cubics (our space no. 2) a method with second order accuracy (in the 1-norm on a polygonal domain) is obtained which has less computational complexity than using piecewise quadratics. If we modify space no. 2 in the same manner (keeping $S^{h}$ the same), we obtain a method with second order accuracy in the 1-norm and third order accuracy in the $L^{2}$-norm on smooth domains. The resulting method has the same computational complexity as Zlámal's.

E. G. \& G. Inc.

680 Sunset Road

P. O. Box 1912

Las Vegas, Nevada 89101

1. S. AGMON, Lectures on Elliptic Boundary Value Problems, Van Nostrand Math. Studies, no. 2, Van Nostrand, Princeton, N. J., 1965. MR 31 \#2504.

2. A. BERGER, " $L$ error estimates for finite elements with interpolated boundary conditions," Numer. Math., v. 21, 1973/74, pp. 345-349. MR 49 \#8395.

3. A. BERGER, R. SCOTT \& G. STRANG, "Approximate boundary conditions in the finite element method," Symposia Mathematica, vol. X, Academic Press, New York, 1972, pp. 757-761.

4. J. BLAIR, "Bounds for the change in the solutions of second order elliptic PDE's when the boundary is perturbed," SIAM J. Appl. Math., v. 24, 1973, pp. 277-285. MR 47 \#6104.

5. J. BLAIR, "Error bounds for spline approximation." (Unpublished manuscript.)

6. J. BRAMBLE \& J. NITSCHE, "A generalized Ritz-least-squares method for Dirichlet problems," SIAM J. Numer. Anal., v. 10, 1973, pp. 81-93. MR 47 \#2836.

7. J. BRAMBLE, T. DUPONT \& V. THOMÉE, "Projection methods for Dirichlet's problem in approximating polygonal domains with boundary-value corrections," Math. Comp., v. 26, 1972, pp. 869-879. MR 49 \#8397.

8. J. BRAMBLE \& A. SCHATZ, "Rayleigh-Ritz-Galerkin methods for Dirichlet's problem using subspaces without boundary conditions," Comm. Pure Appl. Math., v. 23, 1970, pp. 653-675. MR $42 \# 2690$.

9. J. BRAMBLE \& A. SCHATZ, "Least squares methods for $2 m$ th order elliptic boundaryvalue problems," Math. Comp., v. 25, 1971, pp. 1-32. MR 45 \#4657.

10. J. BRAMBLE \& M. ZLAMAL, "Triangular elements in the finite element method," Math. Comp., v. 24, 1970, pp. 809-820. MR 43 \#8250.

11. R. CARLSON \& C. HALL, "Ritz approximations to two dimensional boundary value problems," Numer. Math., v. 18, 1971/72, pp. 171-181. MR 46 \#4751.

12. J.-L. LIONS \& E. MAGENES, Problèmes aux limites non homogènes et applications, Vol. 1, Travaux et Recherches Mathématiques, no. 17, Dunod, Paris, 1968. MR 40 \#512.

13. J.-L. LIONS \& J. PEETRE, "Sur une classe d'espaces d'interpolation," Inst. Hautes Études Sci. Publ. Math., no. 19, 1964, pp. 5-68. MR 29 \#2627.

14. G. STRANG, "Approximation in the finite element method," Numer. Math., v. 19, 1972, pp. 81-98. MR 46 \#4677.

15. G. STRANG \& A. BERGER, "The change in solution due to change in domain," Proc. Sympos. Pure Math., vol. 23, Amer. Math. Soc., Providence, R. I., 1973, pp. 199-205.

16. M. ZLAMAL, "On the finite element method," Numer. Math., v. 12, 1968, pp. 394-409. MR 39 \#5074.

17. M. ZLAMAL, "A finite element procedure of the second order of accuracy," Numer. Math., v. 14, 1969/70, pp. 394-402. MR 41 \#1233. 